\title{
A adaptive based routing for MANET
}

\author{
Zhongqiu Li ${ }^{1}$ Hongpeng Wang ${ }^{2}$ \\ ${ }^{1}$ Department of computer science and technology \\ Harbin Institute of Technology Shenzhen Graduate School \\ Harbin, P.R.China
}

\begin{abstract}
Self-selective routing (SSR) protocol is a novel self-selective, self-maintained and fault-tolerant routing protocol for MANET. SSR has some fatal faults, such as high packet collision and only providing low available bandwidth. In this paper, we present a new routing protocol called Self-selection Based Adaptive Routing (SBAR), which is improved from the SSR protocol by using slot back-off delay time, adding Expanding Ring Search mechanism, and designing an adaptive back-off delay time formula. Experimental results on ns-2 shows that the SBAR can reduce the packet collision rate, provide higher available bandwidth and small end to end delay.
\end{abstract}

Keywords: Adaptive, routing, MANET

\section{Introduction}

Mobile Ad hoc network (MANET) ${ }^{[1]}$ is a collection of wireless mobile nodes communicating with each other using multi-hop wireless links without any existing wired network infrastructure, so the network is very flexible. All nodes are equal and there is no central control node and no fixed routers.

In recent years, routing protocols have received a significant amount of attention. $\mathrm{SSR}^{[2][3]}$ is a novel self-selective routing, self-maintained and fault-tolerant routing protocol for wireless ad hoc networks.
SSR protocol takes advantage of the characteristic of broadcast radio on transmission, and the autonomous algorithm, self-selection, to support routing without the overhead of traditional ad hoc route maintenance. Moreover, the protocol routes packets through a dynamically established and nearly optimal path between the source and destination node. But experimental results show that SSR has also some fatal faults, such as high packet collision rate and only providing low available bandwidth ${ }^{[4]}$.

Toward the faults, we design the SBAR protocol based on SSR by using slot back-off delay time, adding Expanding Ring Search mechanism, and design a adaptive back-off delay time formula. Experimental results on ns-2 show that the SBAR can reduce the packet collision rate, provide higher available bandwidth and SBAR forwards packets using more shorter paths providing more small end to end delay.

The remainder of the paper is organized as follows. In section 2, we will analyze the performance of SSR and present scheme to improve it. In section 3, SBAR is described in detail. Section 4 will presents the experimental results of SBAR on ns-2. Section 5 is conclusion.

\section{Self-Selective Routing (SSR)}

\subsection{Self-Selection Algorithm}

Proceedings of the 11th Joint Conference on Information Sciences (2008)

Published by Atlantis Press (C) the authors 
Leader election ${ }^{[5][6]}$ is a fundamental control problem in both wired and wireless systems. For example, in group communication protocols, the election of a new coordinator is required when a group coordinator crashes or departs the system. In paper [1][2], Gilbert G.Chen proposed a self-selection algorithm, which introduced the leader election algorithm to settle the problem of selecting the forward node among neighbor nodes.

Using self-selection algorithm, when a node wants to send or forward a packet, the node only broadcasts the packet. Receiving the packet, all neighbors count a back-off time using some strategies, then forwards the packet after the delay time. If one node received the packet again during the back-off time, it drops the packet. Then the node with the minimum delay will forward the packet.

Here, we assume that the propagation delay of the signal to each node is negligible. Hence, the nodes are synchronized without the use of any explicit and potentially costly. The backoff delay can not only avoid packet collision, but also provide a precious opportunity to prioritize the status of different nodes, providing a simple solution to packet's forwarding problem.

\subsection{Self-Selective Routing (SSR)}

SSR protocol uses a simple data structure. Each node maintains a destination node cost table, which consist of following items:

- The identity of a destination node

- The sequence number of the last packet from the destination node

- The hop distance to the destination

In SSR, route discovery procedure is divided into route request and route reply procedure and only use the simple flooding to send route request (RREQ) packet during the routing discovery. In the route reply procedure and the data transmission, it uses self-selection algorithm to forwarding packets.

For the back-off time, Chen only gives a simple strategy as following ${ }^{[1]}$.

$$
d_{\text {ackofof }}= \begin{cases}\lambda \times\left(h_{\text {able }}-h_{\text {expocted }}\right) \times U(0,1) & \text { if } h_{\text {able }}>h_{\text {expocted }} \\ \frac{\lambda}{h_{\text {able }}-h_{\text {expected }}} U(0,1) & \text { if } h_{\text {able }} \leq h_{\text {expected }}\end{cases}
$$

In formula (1), $h_{\text {table }}$ is the known number of hops to the destination node, $h_{\text {expected is the number of expected hops }}$ indicated in the route reply (DREP) packet, and $U(0,1)$ is a random number generator producing numbers uniformly distributed over the range defined by its arguments. $\lambda$ is a tuning parameter that must be carefully chosen. If $\lambda$ is too small, the difference between $d_{\text {backoff }}$ calculated by various nodes will be too small to avoid collisions. However, a large $\lambda$ would increase the end-to-end packet delivery delay. As indicated by formula (1), the formula assigns a backoff delay larger than $\lambda$ to nodes with a hop count larger than $h_{\text {expected }}$. The smaller $h_{\text {table }}$ is, the smaller $d_{\text {backoff }}$ will be, and the more likely the node will succeed in transmitting the packet.

\subsection{Self-Selective Routing (SSR)}

SSR is a novel routing protocol for wireless ad hoc networks, with many advantages, self-selective routing, selfmaintained and fault-tolerant etc. But at the same time, there are some disadvantages and the performance of SSR can be increased in some respects.

Firstly, Chen only used the simple flooding to send RREQ during the routing discovery. This algorithm does not scale well to large networks, because

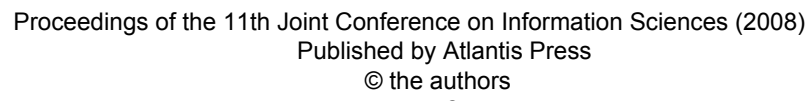


RREQ packets flooded throughout the network may cause a high packet collision rate or even broadcast storm. In SBAR we will use the Expanding Ring Search $^{[7]}$ strategy to reduce the Broadcast Storm Problem ${ }^{[8]}$ in terms of the number of control packets and the level of medium contention in the network.

Secondly, $d_{\text {backoff }}$ is a continue time. As we know, the continue time may add the probability of packet collisions. In SBAR, we use the slot time for back off strategy to reduce the probability of packet collisions.

Thirdly, through NS2 simulation experiment to SSR, we found that the SSR protocol's performance is related to many factor, for example, node's energy, velocity, distance from the last hop etc. From formula (1) we know, SSR only uses the hop distance to compute the back-off delay in self-selection. In SBAR, we will take care of more important parameters to compute $d_{\text {backoff }}$. Those will be explained in detail at next section.

\section{Self-Selection Based Adaptive Routing}

In $\mathrm{SBAR}$, route discovery procedure is divided into route request and route reply procedure. In the route request procedure, we use only the simple flooding with Expanding Ring Search ${ }^{[7]}$ strategy. In the route reply procedure and the data transmission, we will apply self-selection algorithm to improve a new forward method based back-off forwarding, in which the slot delay time will be applied, and we will take into account more factors affecting the performance of SBAR in computing the delay time.

In SBAR, each node maintains a destination node cost table as SSR which was described in 2.2

The detail of SBAR is as follows:

\subsection{Route Request}

In SSR, RREQ packets are flooded throughout the network, so this algorithm does not scale well to large networks. If the destination node is located relatively near the source, issuing a RREQ packet that potentially pass through every node in the network is wasteful. The optimization function is the expanding ring search algorithm, which works as follows.

At first, the source only searches a small area, then searches successively larger areas until the destination node is found. For expanding the "search ring" in which the source is centered, we can only incrementing the time to live (TTL) value carried in every RREQ packet.

\subsection{Route Reply}

Unlike the RREQ packet, the RREP packet does not rely on flooding to find its return path back to the source. Neither does it use an existing path determined during the traversal of the RREQ packet. The destination node simply broadcasts the RREP packet without specifying the next hop. It obtains the hop count to the source from its destination node cost table, then subtracts 1 from it, and puts the result into the expected hop count field in the RREP packet. Every node that detects the arrival of a RREP packet will first inspect its expected hop count field. Now deciding the next hop becomes a self-selection problem.

The central idea of SBAR protocol is to derive the back-off delay based on the known distance, measured by the number of intermediate hops from the destination node, the residue energy of the node, signal strength when node received the RREP packet, buffer threshold of the cache buffer, and the node mobility. This idea is based on the rationale that the nodes which are closer to the destination or have more energy, or have more free buffer etc, should be given higher priority to forward the packet than other nodes.

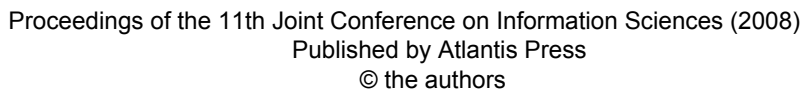


However, by passively listening to all packets and looking into the actual hop count field, an intermediate node only knows the distance from the destination node to itself, not the opposite direction. Unidirectional link in the forward path may result in a longer return path.

We compute the slot back-off delay time $d_{\text {slot }}$ according to the equations (2) follows:

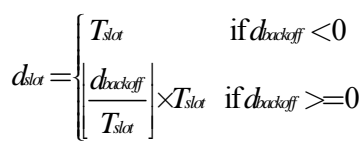

In formula (2), $d_{\text {backof }}$ is the consecutive delay time which is defined by formula (3), which is transformed to slot time of $d_{\text {slot }} . T_{\text {slot }}$ is one slot of time.

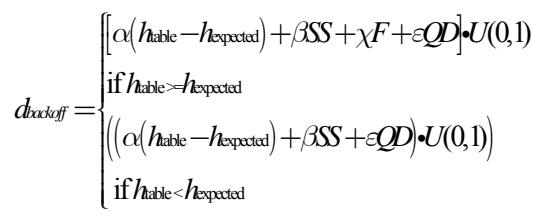

In formula (3), $h_{\text {table is }}$ the known number of hops to the destination node (available from the current node cost table), expected is the number of expected hops carried by the RREP packet, and $U(0,1)$ is a random number generator producing numbers uniformly distributed over the range defined by its arguments. Parameters $\alpha, \beta, \chi$ and $\varepsilon$ are some tuning parameters.

$S S$ is an integer transformed from the signal strength when node received the packets. $S S$ is defined by formula (4).

$$
S S=(\text { int })(p r \times 1 e 10)
$$

In (4), pr is the signal strength $F$ is transformed from the forward rates of packets which have the same destination
ID as current packets. In formula (5), forward represents the number of packets forwarded to the destination when received represents the number of received packets by node whose destination is the same.

$$
F= \begin{cases}0 & \text { if received }=0 \\ 10-\frac{10 \times \text { forward }}{\text { received }} & \text { if received }>0\end{cases}
$$

$Q D$ is the density of the back-off queue in node, which is used to cache the back-off packets.

\subsection{Data Transmission}

Upon receiving a RREP packet, the source can start transmitting DATA packets towards the destination. DATA packets are transmitted and treated in the same way as RREP packets, as both use an actual hop count field and selfselection for forwarding. Therefore, upon the receipt of either a DATA or RREP packet, the receiving node can update the entry in its destination node cost table corresponding to the node from which the packet originated.

\section{Performance evaluation}

To understand and evaluate the effectiveness of our proposed SBAR protocol, we have implemented SSR and SBAR protocol under NS-2(version 2.31) Several sets of simulations have been conducted to compare between SSR, SBAR protocols.

We use IEEE 802.11 as the MAC protocol. The transmission range is 250 meters and the radio propagation model is TwoRayGround model. 100 nodes are placed uniformly in a rectangular field of $2000 \mathrm{~m} \times 2000 \mathrm{~m}$ and TCP traffics with packet size of 1024 bytes are used. The speed of nodes is uniformly varied

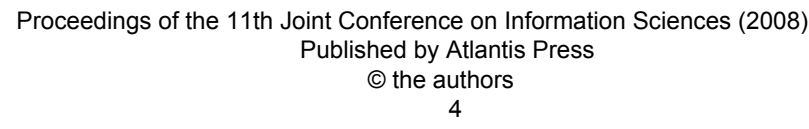


between $10 \mathrm{~m} / \mathrm{s}$ to $100 \mathrm{~m} / \mathrm{s}$ sec with average interval of $2 \mathrm{~s}$, which is another measure of mobility.

The end to end delay of SSR and SBAR are compared for different packet rates. The simulation results of delay changes with packets sending rates can be shown in Figure 1.

Figure 2 shows the performance comparison of SBAR and SSR on packets drop ratio and average delay. The SBAR performs better than SSR for all different packet rates. When the packet rate is 40 (pkts/s), the delay of SBAR is $0.1 \mathrm{~s}$ and $0.4 \mathrm{~s}$ for SSR, the latter is $300 \%$ higher than the former. With the packets sending rate increases above 70 (pkts/s), both of the delay decrease, but the delay of SBAR increases much slower than SSR. So the SBAR performs better than SSR in the point of packet delay. And the drop rate of SBAR is better than SSR also.
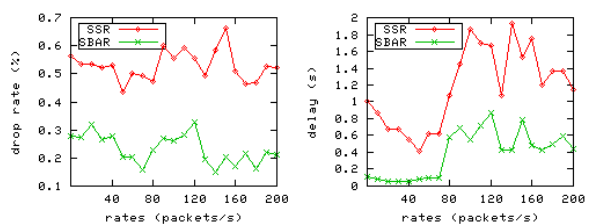

Figure 1. drop rate and packets delay

Figure 2 shows the performance comparison of SBAR and SSR on average throughput of the network and routing cost. The performances of SBAR are almost better than SSR. So in brief, our proposed protocol is efficient than SSR.
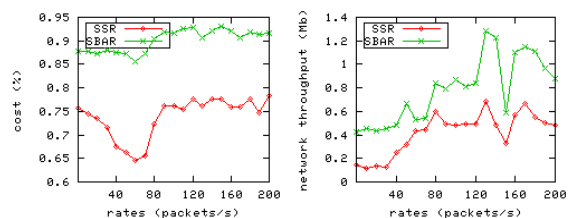

Figure 2. network throughput and routing cost

\section{Conclusions}

In this paper, we proposed the SBAR at base of SSR protocol which by using
ERS strategy to reduce the Broadcast Storm Problem and using slot back off time and using some factor which is relative with protocol's performance to compute delay time. Using ns-2 simulator, we compared our routing scheme to SBAR and SSR, and observed that our technique resulted in a enormous reduction in packet delay, routing cost, and packets drop rate at the same time the network throughput is increased.

\section{References}

[1] J. P. Macker, M. S. Corson, "Mobile Ad Hoc Networking and the IETF", Mobile Computing and Communications Review, 1998, 2(1): 9-14.

[2] G. Chen, J.W. Branch, B.K. Szymanski, "Self-selective routing for wireless ad hoc networks", Proc. of IEEE Int. Conf. Wireless and Mobile Computing, 2005, vol. 3, 5765.

[3] G. Chen, J.W. Branch, B.K. Szymanski, "A self-selection technique for flooding and routing in wireless ad-hoc networks", Journal of Network and Systems Management, 2006, 14(2):359-380.

[4] Jain M., Dovrolis C., "End-to-End available bandwidth: Measurement methodology, dynamics, and relation with TCP throughput", IEEE/ACM Trans.on Networking (TON), 2003, 11(4):537-549.

[5] J. Villadangos, A. Cordoba, F. Farina, M. Prieto, "Efficient leader election in complete networks Villadangos", 13th Euromicro Conference on 9-11 Feb, 2005, 136-143.

[6] Maria Castillo, Federico Farina, Alberto Cordoba, Jesus Villadangos, "AModified $\mathrm{O}(\mathrm{n})$ Leader Election Algorithm for Complete Networks", Parallel, Distributed and NetworkBased Processing, 2007. 
[7] Pham N. D., Choo H., "Energy

Efficient Expanding Ring Search for Route Discovery in MANETs", ICC '08. IEEE International Conference, 2008, 3002-3006 .

[8] Sze-Yao Ni, Yu-Chee Tseng, YuhShyan Chen, and Jang-Ping Sheu, "The broadcast storm problem in a mobile ad hoc network", Proceedings of the fifth annual ACM/IEEE international conference on Mobile computing and networking, ACM Press, 1999, pages 151-162. 